\title{
A first comparison between LIGO and Virgo inspiral search pipelines
}

\author{
F Beauville ${ }^{1}$, M-A Bizouard ${ }^{2}$, L Blackburn ${ }^{3}$, L Bosi $^{4}$, P Brady $^{5}$, \\ L Brocco ${ }^{6}$, D A Brown ${ }^{5,7}$, D Buskulic ${ }^{1}$, S Chatterji ${ }^{7}$, N Christensen ${ }^{8}$, \\ A-C Clapson ${ }^{2}$, S Fairhurst ${ }^{5}$, D Grosjean ${ }^{1}$, G Guidi ${ }^{9}$, P Hello ${ }^{2}$, \\ E Katsavounidis ${ }^{3}$, M Knight ${ }^{8}$, A Lazzarini ${ }^{7}$, F Marion ${ }^{1}$, B Mours ${ }^{1}$, \\ F Ricci $^{6}$, A Viceré ${ }^{9}$ and M Zanolin ${ }^{3}$ (The joint LIGO/Virgo working \\ group) \\ ${ }^{1}$ Laboratoire d'Annecy-le-Vieux de physique des particules, Chemin de Bellevue, BP 110 , \\ 74941 Annecy-le-Vieux Cedex, France \\ ${ }^{2}$ Laboratoire de l'Accélérateur Linéaire (LAL), IN2P3/CNRS-Université de Paris-Sud, B.P. 34, \\ 91898 Orsay Cedex, France \\ ${ }^{3}$ LIGO-Massachusetts Institute of Technology, Cambridge, MA 02139, USA \\ ${ }^{4}$ INFN Sezione di Perugia and/or Università di Perugia, Via A. Pascoli, I-06123, Perugia, Italy \\ ${ }^{5}$ University of Wisconsin-Milwaukee, Milwaukee, WI 53201, USA \\ ${ }^{6}$ INFN, Sezione di Roma and/or Università 'La Sapienza', P.le A. Moro 2, I-00185, Roma, Italy \\ ${ }^{7}$ LIGO-California Institute of Technology, Pasadena, CA 91125, USA \\ ${ }^{8}$ Carleton College, Northfield, MN 55057, USA \\ ${ }^{9}$ INFN-Sezione Firenze/Urbino Via G.Sansone 1, I-50019 Sesto Fiorentino and/or Università \\ di Urbino, Via S.Chiara 27, I-61029, Urbino, Italy
}

Received 12 April 2005, in final form 17 June 2005

Published 6 September 2005

Online at stacks.iop.org/CQG/22/S1149

\begin{abstract}
This paper reports on a project that is the first step the LIGO Scientific Collaboration and the Virgo Collaboration have taken to prepare for a mutual search for inspiral signals. The project involved comparing the analysis pipelines of the two collaborations on data sets prepared by both sides, containing simulated noise and injected events. The ability of the pipelines to detect the injected events was checked, and a first comparison of how the parameters of the events were recovered has been completed.
\end{abstract}

PACS numbers: $04.80 . \mathrm{Nn}, 07.05 . \mathrm{Kf}, 95.55 . \mathrm{Ym}$

(Some figures in this article are in colour only in the electronic version)

\section{Introduction}

The LIGO Scientific Collaboration and the Virgo Experiment have agreed to pursue a joint search for binary inspiral signals [1]. The proposal defines goals and steps to scientifically analyse data in the search for burst events and for coalescence and mergers of compact binary 

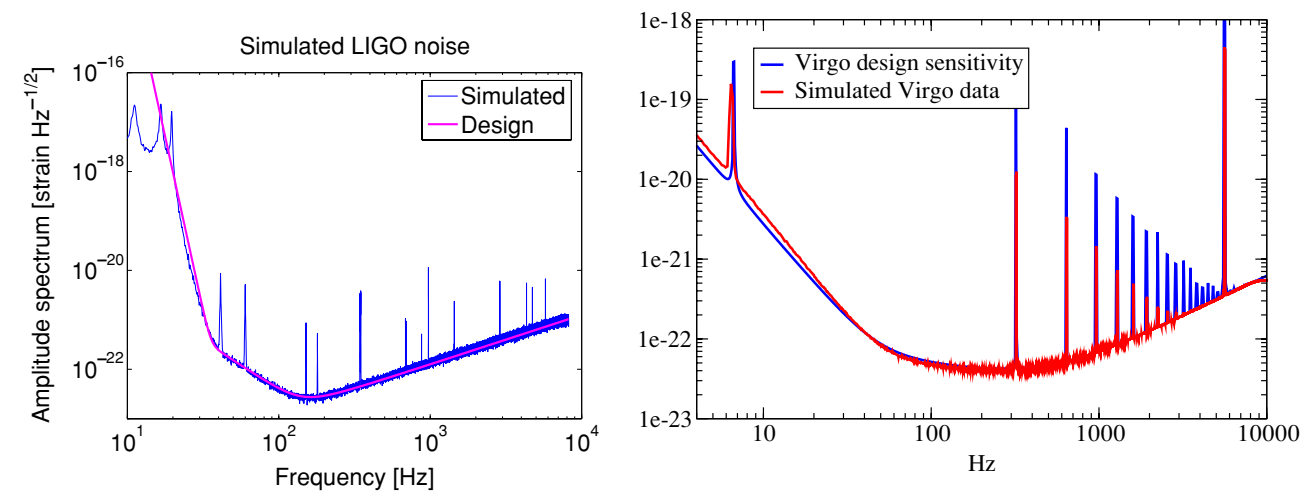

Figure 1. Amplitude spectral density of simulated noise matching the design sensitivity of LIGO (left) and Virgo (right), in strain $/ \sqrt{\mathrm{Hz}}$.

systems. To achieve these goals, both collaborations have undertaken to work together on a series of preparatory data analysis projects of increasing complexity.

This paper reports on the work done on the first test project in the search for inspiral signals from compact binary systems. The primary purpose of the first project was to gain a better understanding of the data analysis algorithms and procedures employed by each group, and to develop a common analysis language.

The first project involved learning how to exchange interferometer data and trigger files, and gaining experience in analysing each other's data. It also involved comparing the various search algorithms and their implementations for detection efficiency, as well as computational efficiency. In particular, we wanted to verify that both groups' detection algorithms identify the same injected events in the data streams and that the recovered parameters of these events are in good agreement with the injection parameters, in order to establish confidence in both our injection and detection procedures. This allowed us to compare alternative implementations of matched filtering for a binary inspiral search. A similar project has been carried out simultaneously for burst events [2].

Each collaboration created three hours of single interferometer simulated data matching their design sensitivity. Additionally, each group provided a series of inspiral injections to be added to the data. Both the LSC and Virgo analysis codes were then run over both data sets, over the same source mass range and with the same threshold on the signal-to-noise ratio. Triggers from the searches have been exchanged, to compare detection efficiency, computational cost and parameter recovery.

The first section of this paper presents the features of the simulated data that have been generated by each collaboration for the project. The analysis pipelines used to search the data are described in section 2. Section 3 is devoted to the trigger production, and section 4 presents a detailed comparison of the events identified by the different analysis codes.

\section{Simulated data}

To serve as a benchmark for this first project, each collaboration produced three hours of single interferometer data consisting of coloured stationary Gaussian noise at their own sample rate (16384 Hz for LIGO and $20000 \mathrm{~Hz}$ for Virgo) with a spectrum matching their design sensitivity, including expected narrowband features. Figure 1 shows the amplitude spectral density of each set of simulated data. 
In addition to the detector noise, each group provided a series of non-coincident, optimally oriented, neutron star inspiral waveforms to be injected in the data. The events were generated using second-order post-Newtonian waveforms.

A number of 26 such events were randomly injected in the LIGO data, with source masses either $\left[1.4 M_{\odot}, 1.4 M_{\odot}\right]$ or $\left[1 M_{\odot}, 2 M_{\odot}\right]$, with $M_{\odot}$ the mass of the Sun. The source was assumed to be located at a distance either $20,25,30$ or $35 \mathrm{Mpc}$ from the detector. The waveforms were generated with a starting frequency of $40 \mathrm{~Hz}$, consistent with the interferometer sensitivity, and injected with an average period of about $400 \mathrm{~s}$.

In the Virgo data, 11 inspiral events with source masses $\left[1.4 M_{\odot}, 1.4 M_{\odot}\right]$ were randomly injected with an average period of about $900 \mathrm{~s}$. The starting frequency of the waveform was $24 \mathrm{~Hz}$, and the events were injected with a signal-to-noise ratio (SNR) of 10, corresponding to a distance of $24.8 \mathrm{Mpc}$ between the source and the detector.

All data were stored in frame format, and only calibrated strain data were exchanged. Details of the injected signals were also exchanged in advance.

\section{Analysis pipelines}

Each group analysed both sets of LIGO and Virgo simulated data, using their own analysis pipeline. In this section we give a short description of the analysis codes used. They are all based on matched filtering implemented on a bank of templates covering some given space for the source masses.

\subsection{LSC pipeline}

A detailed description of the LSC inspiral search pipeline can be found in [3]. We only summarize here the main features of the algorithm.

The data set is split into analysis chunks of 15 overlapping segments. The length of each segment is chosen so as to be at least four times the duration of the longest template in the bank, which depends on the starting frequency of the search. For each analysis chunk, a bank of templates is created, generating templates in the frequency domain using second-order post-Newtonian waveforms.

The data are filtered with each template in the bank, and a trigger is recorded each time the maximum of the SNR of a filter output exceeds some threshold. Maxima above threshold separated in time by less than the length of the template are considered the same trigger, but triggers from different templates are recorded individually.

\subsection{Virgo pipeline}

For this project, the multi-band search (MBTA) [4], which is one of the template-based analyses implemented by the Virgo Collaboration, has been used. In this approach, the templates are split for efficiency into low and high frequency parts and then combined together, in a hierarchical way.

For each frequency band, a bank of 'real' templates is created, generating templates first in the time domain using second-order post-Newtonian waveforms. These templates are called real because they are actually used to filter the data. The analysis is done on data segments long enough to accommodate at least twice the longest template length.

On the full frequency band, a bank of 'virtual' templates is created with the same criteria, but the templates are not used directly in matched filters. For each virtual template, the output of the corresponding filter is built coherently from the outputs of the filters based on the real templates associated with the virtual template in each frequency band. 
Table 1. Common search parameters for the LSC and MBTA pipelines.

\begin{tabular}{lcl}
\hline & LIGO data & Virgo data \\
\hline Mass range & $1-3 M_{\odot}$ & $1-3 M_{\odot}$ \\
Grid minimal match & $95 \%$ & $95 \%$ \\
Starting frequency $f_{\text {low }}$ & $40 \mathrm{~Hz}$ & $30 \mathrm{~Hz}$ \\
Longest template duration & $\sim 45 \mathrm{~s}$ & $\sim 96 \mathrm{~s}$ \\
SNR threshold & 6 & 6 \\
\hline
\end{tabular}

Table 2. Configuration and computing cost of each analysis.

\begin{tabular}{|c|c|c|c|c|}
\hline & $\begin{array}{l}\text { LSC pipeline } \\
\text { on LIGO data }\end{array}$ & $\begin{array}{l}\text { MBTA pipeline } \\
\text { on LIGO data }\end{array}$ & $\begin{array}{l}\text { LSC pipeline } \\
\text { on Virgo data }\end{array}$ & $\begin{array}{l}\text { MBTA pipeline } \\
\text { on Virgo data }\end{array}$ \\
\hline Number of templates & $\sim 3500$ & $\sim 1900$ & $\sim 9500$ & $\sim 6100$ \\
\hline Band splitting frequency & Not relevant & $130 \mathrm{~Hz}$ & Not relevant & $95.3 \mathrm{~Hz}$ \\
\hline Number of jobs & 6 & 1 & 3 & 7 \\
\hline $\begin{array}{l}\text { Type of processor } \\
\text { Total memory }\end{array}$ & $1 \mathrm{GHz}$ Pentium II & $\begin{array}{l}\text { Xeon } 2 \mathrm{GHz} \\
0.9 \text { GByte }\end{array}$ & Xeon $2.66 \mathrm{GHz}$ & $\begin{array}{l}\text { 2.4 GHz Pentium IV } \\
\text { 4.5 GBytes }\end{array}$ \\
\hline $\begin{array}{l}\text { Total processing time } \\
\text { processing time } \times \text { processor speed } \\
\text { number of templates }\end{array}$ & $\begin{array}{l}\sim 46 \mathrm{~h} \\
\sim 47 \mathrm{~s} \mathrm{GHz}\end{array}$ & $\begin{array}{l}\sim 13 \mathrm{~h} \\
\sim 49 \mathrm{~s} \mathrm{GHz}\end{array}$ & $\begin{array}{l}\sim 88 \mathrm{~h} \\
\sim 89 \mathrm{~s} \mathrm{GHz}\end{array}$ & $\begin{array}{l}\sim 28 \mathrm{~h} \\
\sim 40 \mathrm{~s} \mathrm{GHz}\end{array}$ \\
\hline
\end{tabular}

A trigger is recorded each time the maximum of the SNR of a (virtual) filter output exceeds some threshold. The triggers are then clustered both in time and over the template bank; triggers issued by different templates but with matching ending time are considered the same event.

A 'flat search' more similar to the LSC pipeline has also been implemented in Virgo, and was run on both data sets. The triggers obtained are consistent with those resulting by the MBTA analysis and for the sake of paper's clarity we do not report further details. Readers interested in the 'flat search' approach can refer to the paper by Bosi et al in these proceedings [5].

\section{Production of trigger lists}

The LSC pipeline and the MBTA pipeline were used to analyse both data sets, with common search parameters agreed upon beforehand by the two groups. The parameters of the analysis are summarized in table 1 . The source mass space explored was $1-3 M_{\odot}$. The template banks were generated matching a grid in the mass space created with a minimal match criterion of $95 \%$, ensuring that no event in that mass space should be detected with a SNR loss greater than $5 \%$.

The starting frequency $f_{\text {low }}$ used to build the templates was set to different values to analyse the LIGO data and the Virgo data, to be consistent with the SNR accumulation driven by the sensitivity curves of both experiments; $f_{\text {low }}$ was set to $40 \mathrm{~Hz}$ for LIGO data and to $30 \mathrm{~Hz}$ for Virgo data. In addition, the MBTA code was run with a splitting frequency between the low and high frequency bands chosen so as to share in an approximately equal way the SNR between the two bands (see table 2). Triggers were recorded when the SNR exceeded a threshold of 6.

The layout of the template banks depends on the noise power spectral density of the instrument, and on the value chosen for $f_{\text {low }}$. The way the LSC designs the grid used to place the templates is described in [6]. The Virgo group creates the grid according to a 2D contour reconstruction technique based on the parameter space metric [7]. The two methods lead to numbers of templates that differ by about $40 \%$, as is reported in table 2 . 
Table 2 also provides information about the way the production was done for the two pipelines and each data set: on how many jobs the production was split (the LSC pipeline analyses different time periods in different jobs, whereas the MBTA explores different regions of the parameter space), which type of processor was used and how much resources were needed (memory, total processing time after initialization).

In order to compare the performances of the two pipelines, the table also quotes the amount of time required to analyse one template, normalized by the speed of the processor. The speeds of all the analyses are about the same, apart from the LSC pipeline running on Virgo data, presumably because it did not use a number of points which was a power of 2 , and this would have slowed down the fast Fourier transforms. Regarding the MBTA pipeline, the version of the code used was optimized for memory.

\section{Comparison of triggers}

Since both groups use different formats to record their triggers, some specific software was developed to convert triggers from one format to the other.

\subsection{Injection identifications}

The first point was to compare how both groups' detection procedures were able to identify the inspiral injections present in the data streams. In each trigger list, an element is tagged true if the ending time of the event matches the ending time of the injected event within $\pm 20 \mathrm{~ms}$. The detection efficiency and the identification overlap of the two pipelines are very good: The 11 events injected in the Virgo data are detected by both pipelines. In the LIGO data stream, there is one event out of 26 which is missed by both analyses, and one event which is identified by the LSC pipeline but missed by the Virgo pipeline. This is interpreted as a threshold effect since both events were injected at a distance of $35 \mathrm{Mpc}$, leading to an expected low value of 6.40 for the SNR.

An issue was to associate the triggers produced by the LSC pipeline with those produced by MBTA. The LSC triggers are not clustered over the template bank, so that many of them usually correspond to a given injection. On the other hand, MBTA usually produces a single trigger per injection due to the clustering over templates. This becomes important to further compare the detection parameters of events identified by the codes of the two groups. For each injection, the trigger with highest SNR among the LSC associated triggers was kept to be compared to the corresponding MBTA trigger.

\subsection{Signal-to-noise ratio}

For each injected event coincidentally detected by both pipelines, the measured signal-to-noise ratio was compared. The result of the comparison is shown in figure 2. On both data sets, there is a good correlation and general agreement between the SNRs measured by the LSC and MBTA pipelines.

In the case of the Virgo data, the two pipelines appear to be slightly biased in opposite directions, so that there is an average $8 \%$ discrepancy between the measured SNRs. The statistically significant part of the difference can be attributed to differences in the way the template grids are generated. In particular, while the LSC grid has a point very close to $\left[1.4 M_{\odot}, 1.4 M_{\odot}\right]$, the grid used by MBTA does not have a template very close to this point where signals were injected. The closest template has a match only slightly above the $95 \%$ required minimal match. 

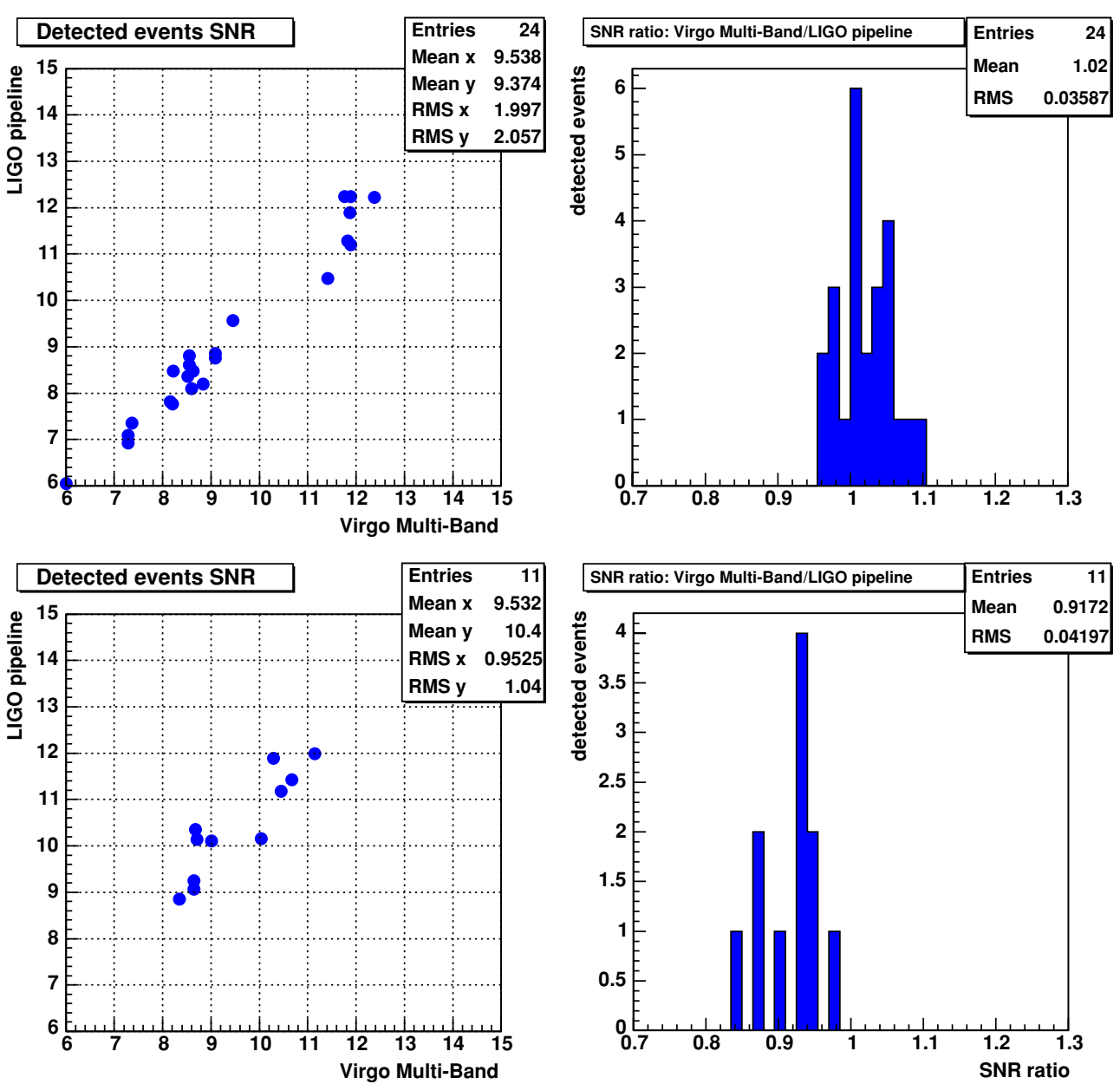

Figure 2. Comparison of the measured signal-to-noise ratio. The top row corresponds to the LIGO produced data, while the bottom row is Virgo produced.

\subsection{Distance}

The distances to the source measured by both pipelines were also compared, as shown in figure 3. As expected, the same correlation and general agreement as in the SNR case is observed. The quality of the agreement is somewhat degraded, however, and in the worst case of the Virgo data, is at the $12 \%$ level.

The systematic effects at the origin of this bias in the distance recovery are yet to be investigated. In the MBTA case, for instance, it is clear that the distance overestimation exceeds the loss in the measured SNR, which is not consistent. The way triggers from the two pipelines are associated for comparison could also have an impact.

\subsection{Arrival time}

A crucial parameter in view of coincident analysis is the arrival time of the detected events. It is essential that the two pipelines agree on this parameter. Figure 4 shows a comparison of the arrival times quoted by the LSC and MBTA pipelines. 

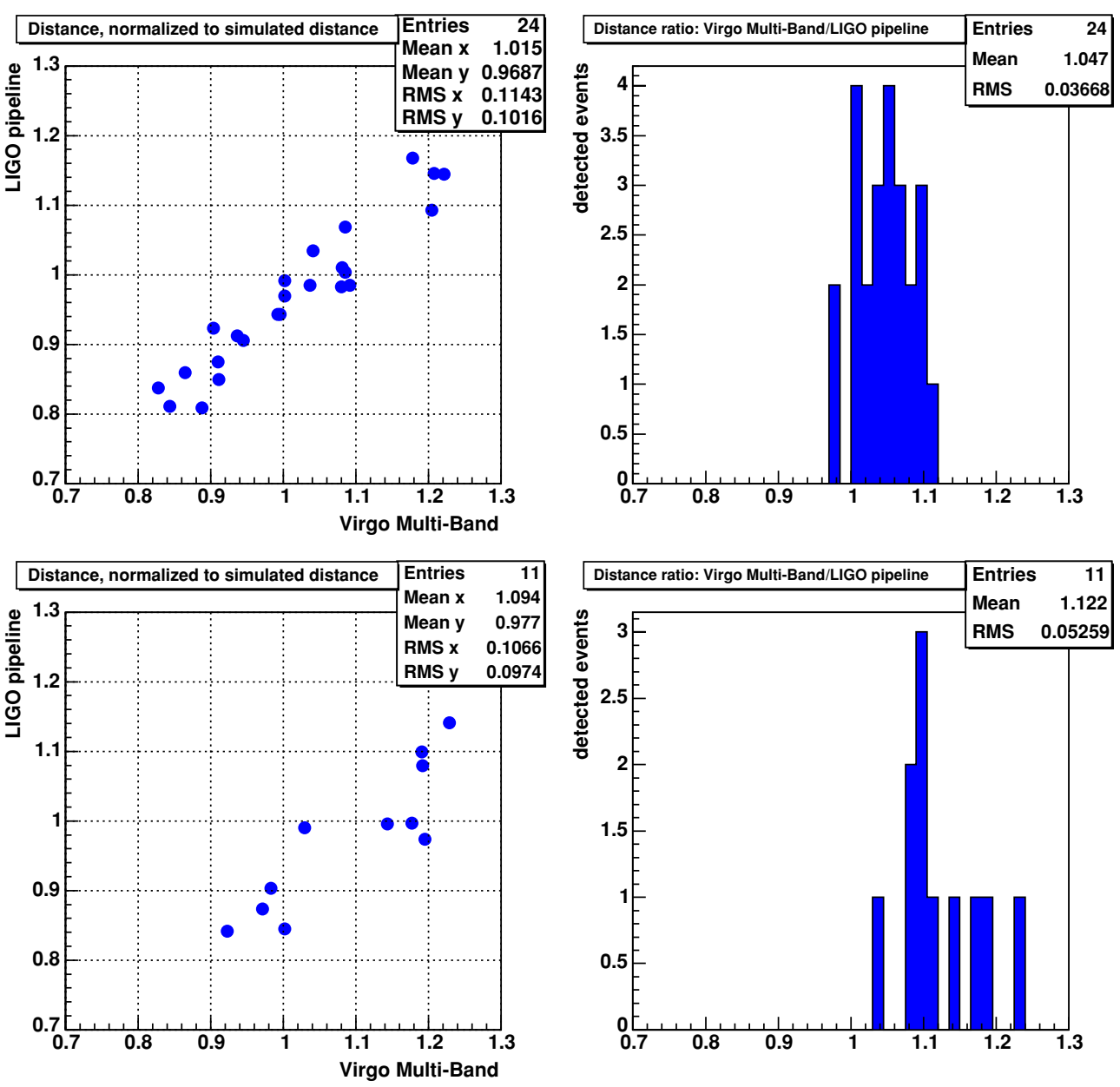

Figure 3. Comparison of the measured source distance, normalized to the simulated distance. The top row corresponds to the LIGO produced data, while the bottom row is Virgo produced.

Interestingly, the plots exhibit some correlation also for the arrival time. In the case of LIGO data, the MBTA pipeline is clearly off for two events detected by the same template which does not behave well as far as the arrival time is concerned. Apart from these two events, which are detected with a low SNR, the agreement between the measured arrival times is at the $1 \mathrm{~ms}$ level.

\subsection{Source mass}

The last test was to see how accurately the mass parameters of an injected inspiral signal could be reconstructed. Figure 5 shows that neither pipeline is able to obtain the component masses particularly accurately. On the other hand, a reliable estimate of the chirp mass, $m_{\text {chirp }}=\left(m_{1} m_{2}\right)^{3 / 5} /\left(m_{1}+m_{2}\right)^{1 / 5}$, where $m_{1}$ and $m_{2}$ are the masses of the two neutron stars, can be obtained. For the two data sets, we see that the accuracies of both pipelines are comparable. Furthermore, we recover the chirp mass very well-errors less than $4 \times 10^{-3}$ in LIGO data and $1 \times 10^{-3}$ in Virgo data (in contrast to previous sections, the two numbers 

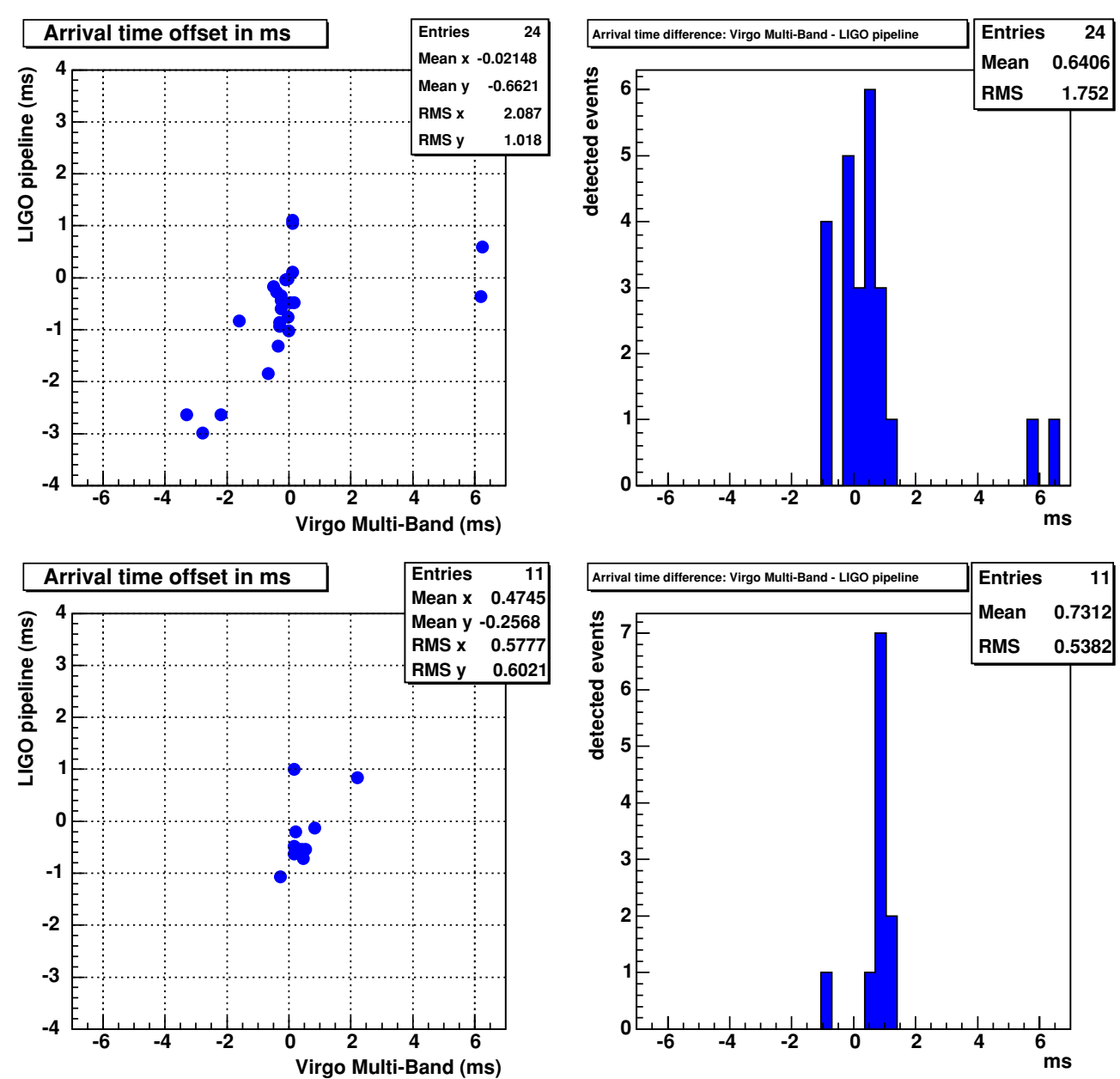

Figure 4. Comparison of the measured arrival time. The top row corresponds to the LIGO produced data, while the bottom row is Virgo produced.

we quote here are based on the different data sets rather than the different pipelines, which actually give comparable results). Thus, the chirp mass seems a natural parameter to use in coincidence tests. The better accuracy in Virgo data is due to better low frequency sensitivity and hence longer waveforms. The errors in the chirp mass appear uncorrelated between the pipelines, and are probably due to the specific template placement algorithms used. Regarding $\eta=m_{1} m_{2} /\left(m_{1}+m_{2}\right)^{2}$, the reconstruction accuracy is intermediate between the individual masses and the chirp mass, typically at the level of a few per cent.

As a post-processing on selected data sections, a Bayesian parameter estimation routine was also applied to the sections of data where the inspiral triggers were found. A Markov chain Monte Carlo (MCMC) routine using a Metropolis-Hastings algorithm generated estimates for the posterior probability distribution functions (PDFs). This method, which is designed to find 2.5 post-Newtonian inspiral signals, is described in $[8,9]$. Figure 6 shows examples of the MCMC produced posterior PDFs for the chirp mass for two of the signals analysed (at an effective distance of $25 \mathrm{Mpc}$ ). For the LIGO produced signal with $m_{1}=1 M_{\odot}, m_{2}=2 M_{\odot}$ (chirp mass $=1.2167 M_{\odot}$ ) the posterior PDF for the chirp mass overlaps the injected value. 

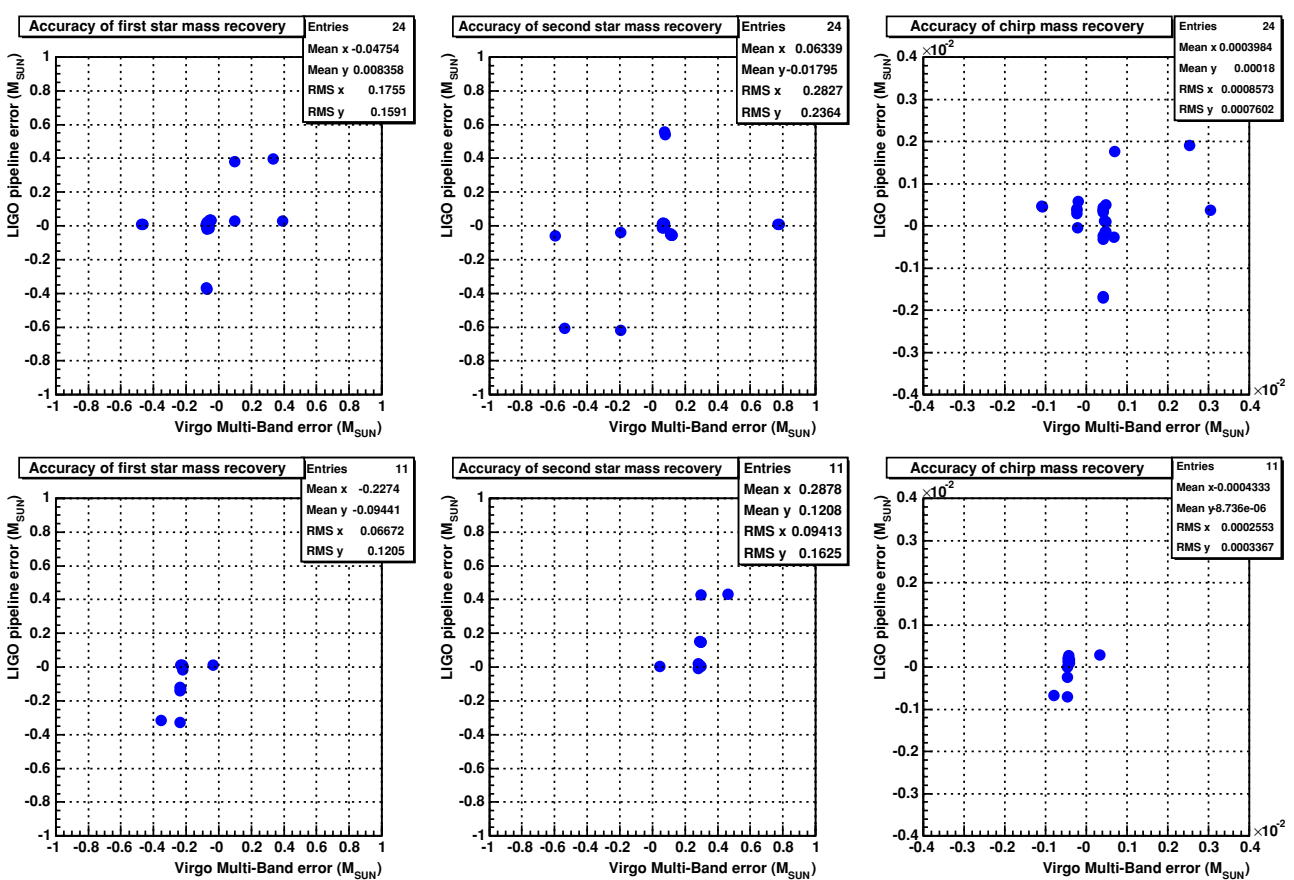

Figure 5. Comparison of the measured source mass parameters. The top row corresponds to the LIGO produced data, while the bottom row is Virgo produced.
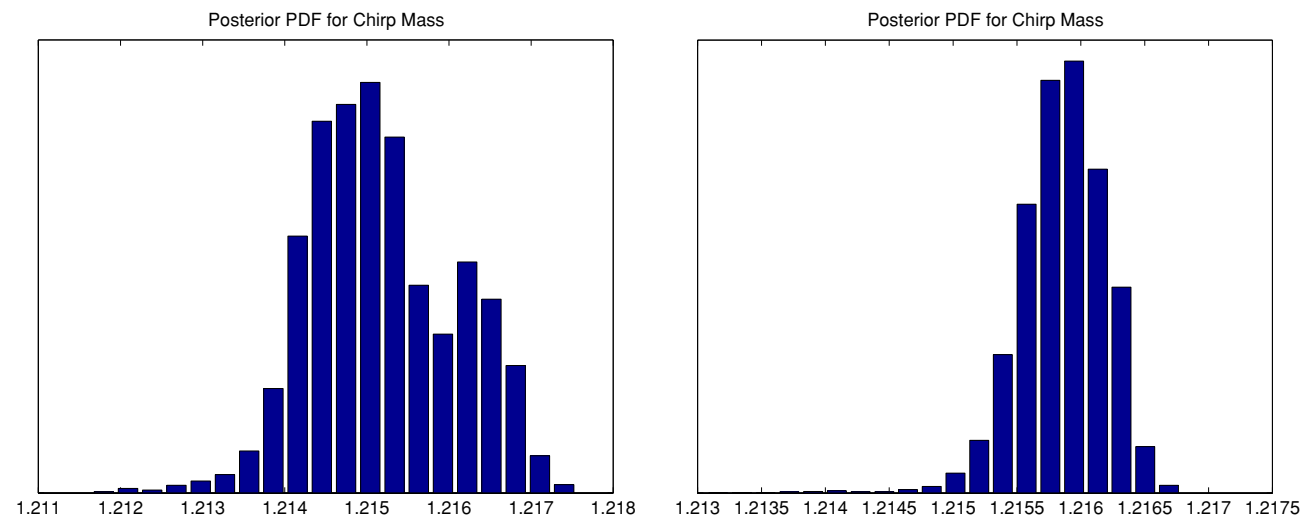

Figure 6. Two examples of posterior PDFs produced by the MCMC for the chirp mass (given in units of solar mass), for sources at an effective distance of $25 \mathrm{Mpc}$. Left: LIGO produced signal at $\left[1 M_{\odot}, 2 M_{\odot}\right]\left(\right.$ chirp mass $\left.=1.2167 M_{\odot}, \eta=0.2222\right)$, PDF mean $=1.2152$, RMS $=$ 0.298 . Estimated $\eta=0.224$. Right: Virgo produced signal at $\left[1.4 M_{\odot}, 1.4 M_{\odot}\right]$ (chirp mass $=$ $\left.1.2188 M_{\odot}, \eta=0.25\right)$, PDF mean $=1.2158$, RMS $=0.185$. Estimated $\eta=0.246$.

For the Virgo produced signal with $m_{1}=m_{2}=1.4 M_{\odot}$ (chirp mass $\left.=1.2188 M_{\odot}\right)$ the mean of the posterior PDF for the chirp mass differs by $0.23 \%$ from the injected value. This slight difference, also seen with the LIGO produced signals with $m_{1}=m_{2}=1.4 M_{\odot}$, is likely due to the different nature of the signals; the generated signals were 2.0 post-Newtonian in the time domain, while the MCMC searches for 2.5 post-Newtonian in the frequency domain. The fact 
that there is not a complete one-to-one match between these two signals is reflected in these estimates.

\section{Conclusion}

In this first project based on simulated data, the LIGO-Virgo joint working group has had the opportunity to run analysis pipelines from the two collaborations on data sets produced by both sides. In doing so, it has been possible to gain better understanding and-most importantly-confidence in each other's detection procedures, since both analysis pipelines have been shown to detect the same events with comparable parameters. This project has thus established the grounds for future work towards collaborative data analysis in the search for inspiral gravitational wave signals.

\section{Acknowledgment}

LIGO Laboratory and the LIGO Scientific Collaboration gratefully acknowledge the support of the United States National Science Foundation for the construction and operation of the LIGO Laboratory and for the support of this research.

\section{References}

[1] Proposal for joint LIGO-Virgo data analysis 2004 LIGO-T040137-08-Z and VIRGO-PLA-DIR-1000-201

[2] Beauville F et al (Joint LIGO/Virgo working group) 2005 Class. Quantum Grav. 22 S1293

[3] Abbott B et al (LIGO Scientific Collaboration) 2004 Phys. Rev. D 69122001

[4] Marion F et al (Virgo Collaboration) 2004 Gravitational waves and experimental gravity Proc. Rencontres de Moriond 2003

[5] Acernese F et al (Virgo Collaboration) 2005 Class. Quantum Grav. 22 S1139

[6] Owen B J and Sathyaprakash B S 1999 Phys. Rev. D 60022002

[7] Buskulic D et al 2003 Class. Quantum Grav. 20789

[8] Christensen N, Meyer R and Libson A 2004 Class. Quantum Grav. 21317

[9] Christensen N and Meyer R 2001 Phys. Rev. D 64022001 